\title{
Novel Synthesis and Functionalization of Boron Nitride Nanotubes
}

\author{
Y. Bando, C. Y. Zhi, Q. Huang, C. C. Tang, and D. Golberg
}

Advanced Materials Laboratory, National Institute for Materials Science (NIMS), Namiki 1-1,Tsukuba, Ibaraki 305-0044, Japan. Email: bando. yoshio@nims.go.jp

Boron nitride nanotube (BNNT) is promising because it has a constant band gap, and exhibit excellent mechanical properties, high thermal conductivity and superb resistance to oxidation [1-2]. Therefore, BNNTs are thought to be primarily useful as a nanoscale semiconductors working in hazardous environments or an additive in composites. However, to date, many unanswered questions, such as adjustment of BNNT's electronic structure, dispersibility of BNNT in a solvent and high-yield production of clean BNNTs etc., have hampered the expecting breakthroughs in the above-mentioned fields.

Here we report highly pure BNNTs were synthesized at high yield via a carbon-free chemical vapor deposition method. Optimized precursor was adopted so that highly pure BNNTs can be obtained in a large temperature range $\left(1100{ }^{\circ} \mathrm{C}\right.$ to $\left.1700{ }^{\circ} \mathrm{C}\right)$ [3-4]. For the first time, BNNTs were perfectly dissolved in various organic solvents by wrapping them with a polymer or covalent functionalization. The functionalized BNNTs were investigated by cathodoluminescence and UV-vis absorption experiments. The results reveal drastic changes in the band structure of BNNTs and strong interaction between BNNTs and the polymer. The biology applications of BNNTs were also explored by immobilizing various proteins on BNNTs [4-9]. Otherwise, peeled BNNTs and BNNT Y-junctions were obtained through chemical reaction in DMSO [10].

\section{References}

1. D. Golberg, Y. Bando, et al., Scripta. Materialia. 44 (2001) 1561.

2. C. Tang, Y. Bando, et al., J. Am. Chem. Soc. 127 (2005) 6552.

3. C. Tang, Y. Bando, et al., Chem. Commun. (2002) 1290.

4. C. Y. Zhi, Y. Bando, et al., Solid State Commun. 135 (2005) 67.

6. C. Y. Zhi, Y. Bando, et al., Angew. Chem. In. Ed. 44 (2005) 7929.

7. C. Y. Zhi, Y. Bando,et al., Angew. Chem. In. Ed. 44 (2005) 7932.

8. C. Y. Zhi, Y. Bando, et al., J. Am. Chem. Soc. 127 (2005) 15996.

9. C. Y. Zhi, Y. Bando, et al., J. Am. Chem. Soc. 127 (2005) 17144.

10. Q. Huang, Y. Bando, Angew. Chem. In. Ed. in press. 

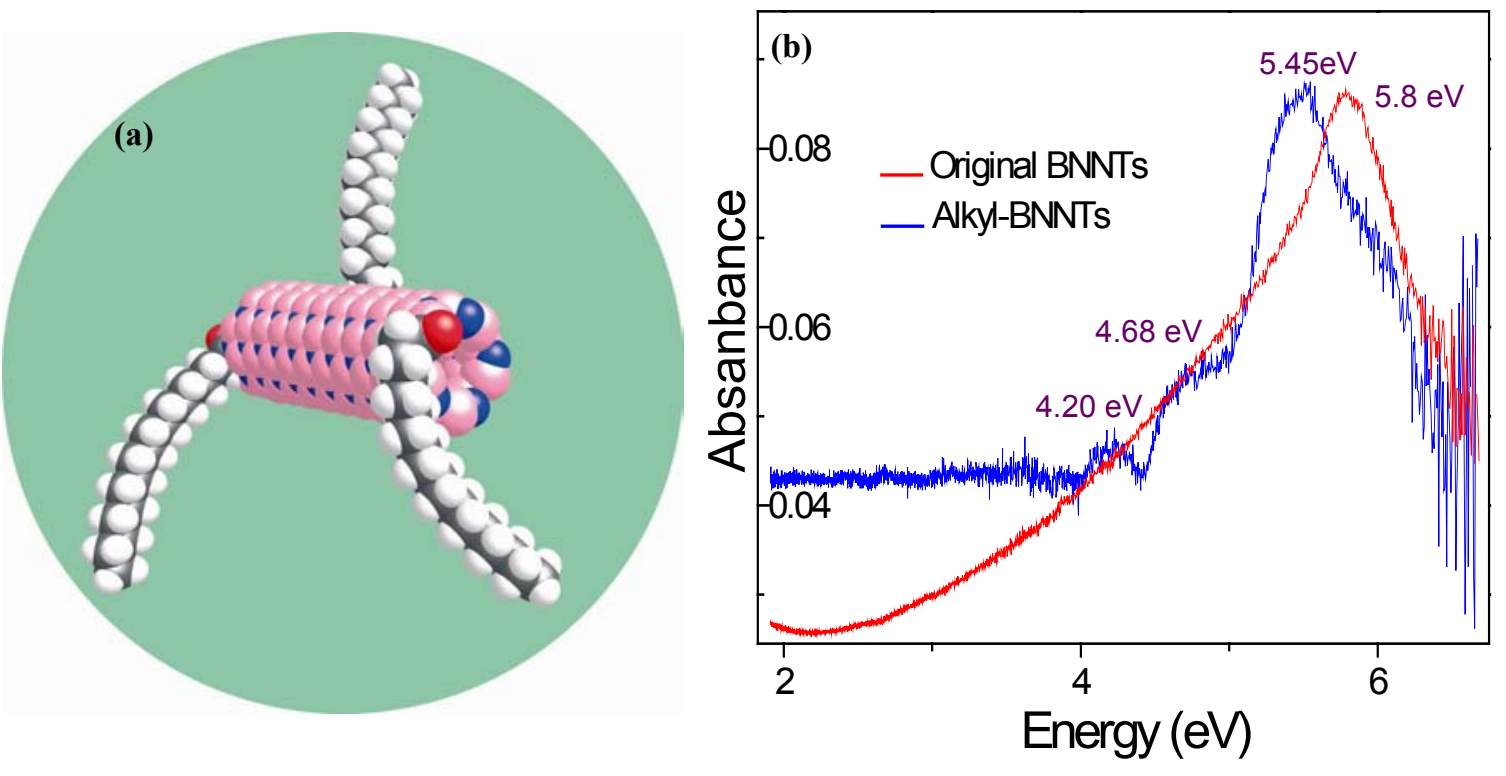

Fig. 1 (a) Schematic diagram of BNNT connected by alkyl chain; (b) UV-vis absorption spectra of original BNNT and alkyl-BNNT.

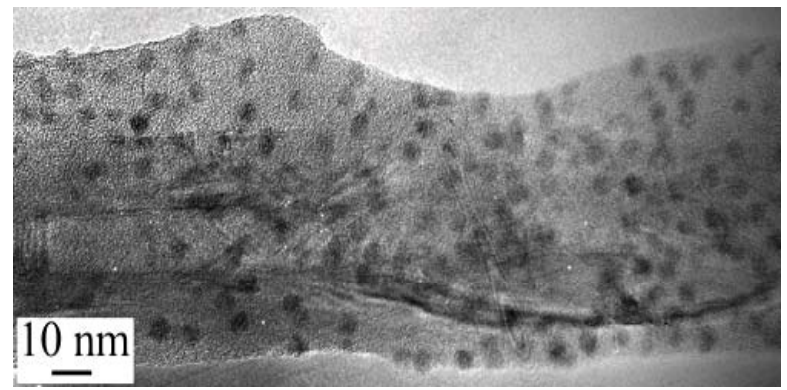

Fig. 2 Ferritin molecules immobilized on a PAHE-functionalized BNNT.

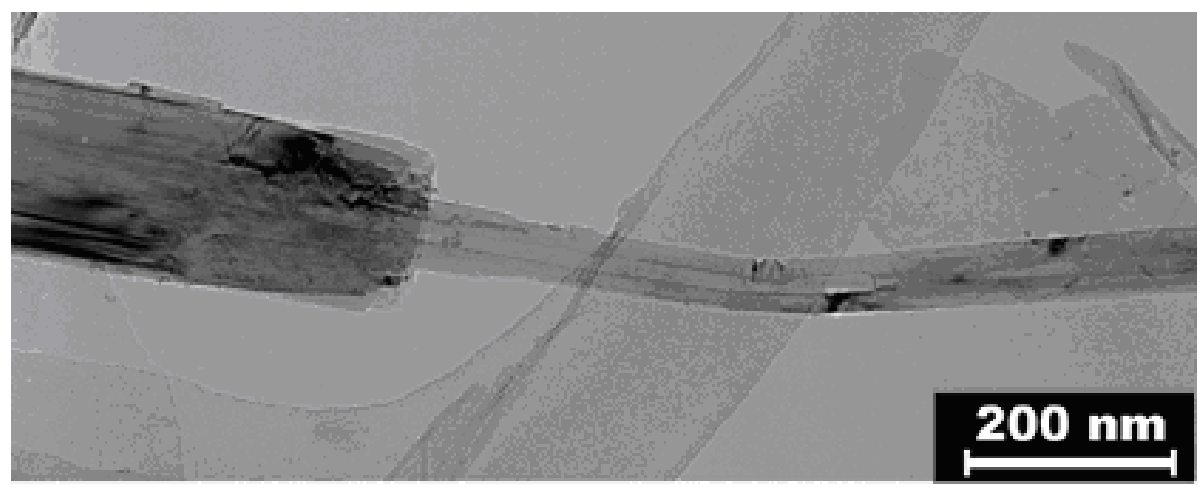

Fig. 3 a Peeled BNNT. 